\title{
Neutrophil-to-lymphocyte ratio in thyroid ophthalmopathy
}

\author{
Celik T \\ Department of Ophthalmology, Bulent Ecevit University Faculty of Medicine, Zonguldak, Turkey. \\ drtubacelik@yahoo.com
}

\begin{abstract}
PURPOSE: To evaluate the neutrophil-to-lymphocyte ratio (NLR) levels to predict the severity of inflammation in thyroid ophthalmopathy (TO).

METHODS: Fifty-six patients with TO and 40 healthy subjects were included in this study. TO patients were divided into two groups according to clinical activity score (CAS). Group 1 included 24 active TO patients and Group 2 included 32 inactive TO patients. The thyroid status, white blood cell (WBC), neutrophil, and lymphocyte counts were performed. NLR was calculated by dividing the neutrophil count by the lymphocyte count. RESULTS: The mean age was $53.6 \pm 5.4$ in active TO group, $54.2 \pm 5.6$ in inactive TO group, and $52.7 \pm 5.2$ in the control group. The WBC, neutrophil, lymphocyte and NLR levels were higher in patients with TO than in the control group $(p<0.05)$. A significant difference in NLR was found between the inactive and active TO groups $(p<0.05)$.

CONCLUSION: NLR values were found to be higher in patients with TO than in controls. NLRvalues were also found higher in active TO patients than in inactive TO patients (Tab. 3, Ref. 26). Text in PDF www.elis.sk. KEY WORDS: neutrophil-to-lymphocyte ratio, thyroid ophthalmopathy, Graves' ophthalmopathy, inflammation, biomarker.
\end{abstract}

\section{Introduction}

Thyroid ophthalmopathy (TO) is an autoimmune inflammatory disease which leads to proptosis, conjunctival pain and hyperemia, extraocular muscle involvement and vision loss by affecting the orbital tissues (1). Although the exact mechanism of TO has not been clarified yet, it has been considered to be associated with the orbital infiltration of $\mathrm{T}$ cells which are essentially recognized by thyroid follicle cells and activated with similar antigens in orbital tissues. These activated $\mathrm{T}$ cells secrete many cytokines such as interleukin-1, tumor necrosis factor alpha and interferones (2). While the orbital infiltration is generally mild to moderate, severe ophthalmopathy can be seen in 3-5\% of all cases (3). Hyperthyroidism is commonly accompanied with TO but in some cases hypothyroidism develops due to Hashimoto's disease and euthyroidism can occur (4).

Neutrophil-to-lymphocyte ratio (NLR) has been recently used as a novel inflammatory marker to assess the severity of inflammation (5). The relationship between NLR values and various systemic diseases such as diabetes mellitus, cardiovascular diseases, hypertension and malignancies were studied in the last research $(5,6)$. Moreover, investigations with NLR levels and some ocular

Department of Ophthalmology, Bulent Ecevit University Faculty of Medicine, Zonguldak, Turkey

Address for correspondence: T. Celik, MD, Department of Ophthalmology, Bulent Ecevit University Faculty of Medicine, Zonguldak, Turkey Phone: +90.3722612265 , Fax: +90.3722610264 diseases have been previously reported particularly for the ophthalmology audience (5-7).

In the current study, we aimed to evaluate the NLR levels to assess the inflammatory process in TO patients. To the best of our knowledge, this is the first clinical study to investigate the inflammatory relationship between TO and NLR values.

\section{Materials and methods}

\section{Patients}

This study was approved by the ethics committee and was conducted in accordance with the Declaration of Helsinki. The details of the study were explained to the patients and written informed consents were obtained.

Fifty-six participants with TO and 40 healthy subjects were enrolled in this clinical study. Twenty-four patients had active TO (Group1), and 32 patients had inactive TO (Group 2). Clinical activity was graded according to the Clinical Activity Score (CAS) (Tab. 1). Active TO was defined as CAS $\geq 3$ (8).

Detailed history, including family history, duration and progression of the disease and smoking were obtained. The patients with acute or chronic infection, inflammatory ocular or systemic diseases, ocular surgery or trauma history during the past 12 months, ocular or systemic drug use, steroid use in the past 6 months, malignancy history and hematologic disorders were excluded from the study.

Best corrected visual acuity, anterior and posterior segments examination and intraocular pressure measurement were applied to all participants. 
Tab. 1. Clinical Activity Score (CAS).

Spontaneous retrobulbar pain

Ocular pain with eye movement

Redness of the eyelids

Swelling of the eyelids

Redness of the conjunctiva

Chemosis of the conjunctiva

Swelling of the caruncule

*1 point is given for each item present.

\section{Laboratory analyses}

Complete blood count (CBC) values were obtained from all the participants. Venous blood samples were obtained from the antecubital vein and $\mathrm{CBC}$ measurements were performed by blood cell counter (ABX Pentra DF 120, Horiba, Japan. The white blood cell (WBC), neutrophil, and lymphocyte counts were recorded. The NLR levels were calculated from neutrophil and lymphocyte parameters. Thyroid-stimulating hormone (TSH), free triiodothyronine (FT3) and free thyroxine (FT4) values were also performed to assess the thyroid status of the patients.

\section{Statistical analysis}

All statistical analyses were performed by using the Statistical Package for Social Science version 16.0 software (SPSS, Chicago, IL). Data were expressed as mean \pm standard deviation. The Kolmogorov-Smirnov test was used to analyze normality of the groups. Comparisons of non-parametric values among groups were performed using one-way ANOVA. Comparisons between the groups were performed by using Mann- Whitney U-test. P values less than 0.05 were considered significant.

\section{Results}

Demographic features of participants with active TO, inactive TO and control group are shown in Table 2. The mean age was $53.6 \pm 5.4$ in the active TO group $(n=24), 54.2 \pm 5.6$ years in the inactive TO group $(\mathrm{n}=32)$ and $52.7 \pm 5.2$ in the control group $(n=40)$. There were 11 men $(42 \%)$ and 13 women $(58 \%)$ in the active TO group; 15 men (44\%) and 17 women (56\%) in the inactive TO group; 20 men (50\%) and 20 women (50\%) in the control group. There were no significant differences among groups in terms of age and gender distribution.

The thyroid status was proved to be euthyroid in all of the 40 healthy subjects. Hyperthyroid status was found in 21 of $24 \mathrm{TO}$ patients $(88 \%), 2$ TO patients ( $8 \%$ ) were euthyroid and 1 TO patient (4\%) was hypothyroid in the active TO group while 26 of 32 TO patients ( $81 \%)$ were hyperthyroid, 2 TO patients $(12 \%)$ were euthyroid and 1 TO patient (6\%) was hypothyroid in the inactive TO group ( $\mathrm{p}=0.62, \mathrm{p}=0.46, \mathrm{p}=0.32$, respectively).

The NLR and WBC levels were higher in both TO groups when compared with the control group.

A significant difference in NLR was found between the inactive and active TO groups $(\mathrm{p}=0.012)$. The WBC, neutrophil and lymphocyte levels were also higher in active group than in inactive group ( $p=0.038, p=0.026, p=0.036$, respectively) (Tab. 3).
Tab. 2. The demographic features and laboratory parameters of TO patients and control group (mean $\pm \mathrm{SD}$ ).

\begin{tabular}{lccc}
\hline & $\begin{array}{c}\text { TO patients } \\
(\mathrm{n}=56)\end{array}$ & $\begin{array}{c}\text { Control group } \\
(\mathrm{n}=40)\end{array}$ & $\mathrm{p}$ \\
\hline Age & $53.9 \pm 5.5$ & $52.7 \pm 5.2$ & 0.665 \\
Gender $(\mathrm{M} / \mathrm{F})$ & $26 / 32$ & $20 / 20$ & 0.542 \\
WBC $\left(10^{3} / \mu \mathrm{l}\right)$ & $8.06 \pm 1.72$ & $6.62 \pm 1,55$ & 0.042 \\
Neutrophil $\left(10^{3} / \mu \mathrm{l}\right)$ & $4.48 \pm 1.68$ & $3.64 \pm 1.24$ & 0.037 \\
Lymphocyte $\left(10^{3} / \mu \mathrm{l}\right)$ & $2.18 \pm 0.66$ & $1.84 \pm 0.52$ & 0.048 \\
NLR & $2.12 \pm 0.72$ & $1.74 \pm 0.48$ & 0.039 \\
\hline
\end{tabular}

Tab. 3. The demographic features and laboratory parameters of active and inactive TO groups (mean \pm SD).

\begin{tabular}{lccc}
\hline Variable & Active TO & Inactive TO & $\mathrm{p}$ \\
\hline Age & $53.6 \pm 5.4$ & $54.2 \pm 5.6$ & 0.625 \\
Gender $(\mathrm{M} / \mathrm{F})$ & $11 / 13$ & $15 / 17$ & 0.764 \\
WBC $\left(10^{3} / \mu \mathrm{l}\right)$ & $9.86 \pm 1.84$ & $6.76 \pm 1.68$ & 0.038 \\
Neutrophil $\left(10^{3} / \mu \mathrm{l}\right)$ & $5.98 \pm 1.76$ & $3.71 \pm 1.52$ & 0.026 \\
Lymphocyte $\left(10^{3} / \mu \mathrm{l}\right)$ & $2.38 \pm 0.64$ & $1.89 \pm 0.56$ & 0.036 \\
NLR & $2.72 \pm 1.2$ & $1.98 \pm 0.54$ & 0.012 \\
\hline
\end{tabular}

\section{Discussion}

TO commonly presents during 4 th-5th decades. While the female-to-male ratio is 5-10/1 in Graves' Disease, it is about 2-5/1 in TO. The probability of thyroid ophthalmopathy increased in HLA-B8, HLA-DR3 and HLA-DAW3 haplotypes in the white race (9). As an autoimmune course, antithyroglobulin which is an autoantibody responsible for the disease is directed against thyroid-derived thyroglobulin and attacks extraocular muscles. Mononuclear infiltration and glycosamioglican accumulation leads to the swelling of orbital tissues (10).

IL-17-secreting T cells play a critical role in the pathogenesis of organ-specific autoimmune diseases. These cells stimulate the production of inflammatory cytokines such as IL-1b, IL-6 and TNF-alpha and also promote inflammatory chemokines that induce the production of neutrophils and macrophages (11). Kim et al found the serum levels of Th17 cells in patients with Graves' ophthalmopathy to be higher than in controls and the serum levels of IL-17 in active Graves' ophthalmopathy patients were higher than those in inactive Graves' ophthalmopathy patients. They concluded that serum IL-17 concentration had a significant correlation with the disease activity (12).

In recent years, NLR has been thought to be a simple, inexpensive and easily-reached predictor to evaluate systemic inflammation. This WBC-derived parameter has been used in several tumoral, cardiac, inflammatory and autoimmune diseases (13-15). WBC subgroups play a critical role in inflammation and cytokine release. Tissue damage, stress, and inflammation result in neutrophilia and lymphopenia (16). It was reported that NLR not only evaluates the inflammatory response, it also predicts the prognosis of the disease (13-15).

The relationship between NLR and ocular diseases such as diabetic retinopathy, glaucoma, keratoconus, non-arteritic ischemic optic neuropathy and age-related macular degeneration were studied in literature previously. Ulu et al reported that increased 
NLR level was associated with the presence of diabetic retinopathy and diabetes mellitus. The authors pointed out that the grade, as well as the severity of diabetic retinopathy is also correlated with NLR levels (17). Subsequently, Wang et al studied the NLR and brachial-ankle pulse wave velocity among the diabetic patients without diabetic retinopathy, patients with diabetic retinopathy and healthy subjects. They found out that both parameters were significantly higher in patients with diabetes mellitus and diabetic retinopathy (18).

The relationship between NLR and age-related macular degeneration (AMD) was also discussed by researchers. Ilhan et al studied NLR levels in 81 patients with dry AMD, 84 patients with wet AMD, and 80 healthy age- and sex-matched controls. They found out that patients with AMD had higher NLR values compared with controls, and NLR was correlated with disease severity. Therefore, the authors concluded that NLR may be used as a biomarker of inflammation in AMD (19). In another study, increased NLR value is found to be independently associated with neovascular AMD (20).

Guclu et al studied 42 patients with acute optic neuritis and 40 healthy subjects to investigate the relationship between NLR and acute optic neuritis episodes. They reported that there was a significant relation between NLR and number of episodes; therefore, higher NLR can be a useful marker for predicting recurrent episodes (21).

Non-arteritic ischemic optic neuropathy (NAION) was also evaluated in terms of NLR relationship. The authors recommended NLR as a new inflammatory marker for the assessment of severity of inflammation in NAION patients (22).

Retinal vein occlusion (RVO) is another disease which was researched for investigating the relation between NLR and RVO. In a study on 40 RVO patients and 40 healthy volunteers, it was found out that high NLR values were related to the development of RVO (23).

Karaca et al evaluated NLR in patients with progressive and non-progressive keratoconus and they reported that NLR was higher in patients with progressive keratoconus than in the nonprogressive group and controls (24).

Two consecutive researches about glaucoma and NLR were reported more recently $(25,26)$. In the first study, the authors evaluated the NLR levels in patients with primary open-angle glaucoma and they found a significant difference in NLR levels between POAG and control groups (25). Subsequently, the second study assessed the levels of NLR in patients with pseudoexfoliation syndrome (PEX), pseudoexfoliation glaucoma (PXG) and healthy controls. NLR was found to be higher in both, PEX and PXG groups; therefore, NLR may be useful for predicting the prognosis of PEX patients and progression to PXG (26).

In our study, we investigated the relationship between the NLR levels and the severity of inflammation in TO patients. As far as we know from the outcomes of previous research, NLR was associated with the disease activity in several inflammatory diseases. On the basis of this, we also evaluated the NLR levels both in active and inactive TO patients. Our results provide evidence that NLR values are higher in active TO patients compared to in- active patients. An elevated NLR value is thought to result from high thyroid levels in the active TO group but we did not found a significant difference between the active and inactive TO groups in terms of thyroid status. This is the first study investigating the relationship between NLR and TO in literature. The only limitation of our study is a relatively small sample size.

In conclusion, we suggest that NLR can be a useful, reliable, inexpensive and easily-measured biomarker to predict both TO and the disease activity. Further research based on larger groups of participants will contribute to literature.

\section{References}

1. McAlinden C. An overview of thyroid eye disease. Eye Vis (Lond) 2014; 1: 9 .

2. Prabhakar BS, Bahn RS, Smith TJ. Current perspective on the pathogenesis of Graves' disease and ophthalmopathy. Endocr Rev 2003; 24 : 802-835.

3. Wiersinga WM, Bartalena L. Epidemiology and prevention of Graves' ophthalmopathy. Thyroid 2002; 12: 855-860.

4. Hiromatsu Y, Eguchi H, Tani J, Kasaoka M, Teshima Y. Graves' ophthalmopathy: epidemiology and natural history. Intern Med. 2014; 53 (5): 353-360.

5. Faria SS, Fernandes PC Jr, Silva MJ et al. The neutrophil-to-lymphocyte ratio: a narrative review. Ecancermedicalscience 2016; 10: 702.

6. Guthrie GJ, Charles KA, Roxburgh CS, Horgan PG, McMillan DC, Clarke SJ. The systemic inflammation-based neutrophil-lymphocyte ratio: experience in patients with cancer. Crit Rev Oncol Hematol 2013; 88 (1): 218-230.

7. Yuksel M, Yildiz A, Oylumlu M et al. Novel markers of endothelial dysfunction and inflammation in Behçet's disease patients with ocular involvement: epicardial fat thickness, carotid intima media thickness, serum ADMA level, and neutrophil-to-lymphocyte ratio. Clin Rheumatol 2016; 35 (3): 701-708.

8. Mourits MP, Prummel MF, Wiersinga WM, Koornneef L. Clinical activity score as a guide in the management of patients with Graves' Ophthalmopathy. Clinical Endocrinology 1997; 47: 9-14.

9. Weiler DL. Thyroid eye disease: a review. Clin Exp Optom 2017; 100 (1): $20-25$.

10. Garrity JA, Bahn RS. Pathogenesis of graves ophthalmopathy: Implications for prediction, prevention, and treatment. Am J Ophthalmol 2006; 142: 147-153.

11. Tabarkiewicz J, Pogoda K, Karczmarczyk A, Pozarowski P, Giannopoulos K. The Role of IL-17 and Th17 Lymphocytes in Autoimmune Diseases. Arch Immunol Ther Exp (Warsz) 2015; 63: 435-449.

12. Kim SE, Yoon JS, Kim KH, Lee SY. Increased serum interleukin-17 in Graves' ophthalmopathy. Graefes Arch Clin Exp Ophthalmol 2012; 250 (10): 1521-1526.

13. Bhat T, Teli S, Rijal J et al. Neutrophil to lymphocyte ratio and cardiovascular diseases: a review. Expert Rev Cardiovasc Ther 2013; 11 (1): 55-59.

14. Küçük E, Kocayiğit İ, Günel C, Düzenli H. Neutrophil-to-lymphocyte ratio in occlusive vascular diseases: the literature review of the past 10 years. World J Emerg Med 2016; 7 (3): 165-172. 


\section{5-498}

15. Templeton AJ, McNamara MG, Šeruga B, Vera-Badillo FE, Aneja P, Ocaña A, Leibowitz-Amit R, Sonpavde G, Knox JJ, Tran B, Tannock IF, Amir E. Prognostic role of neutrophil-to-lymphocyte ratio in solid tumors: a systematic review and meta-analysis. J Natl Cancer Inst 2014; 106 (6): dju124.

16. Zahorec R. Ratio of neutrophil to lymphocyte counts-rapid and simple parameter of systemic inflammation and stress in critically ill. Bratisl Lek Listy. 2001; 102 (1): 5-14.

17. Ulu SM, Dogan M, Ahsen A, Altug A, Demir K, Acartürk G, Inan S. Neutrophil-to-lymphocyte ratio as a quick and reliable predictive marker to diagnose the severity of diabetic retinopathy. Diabetes Technol Ther 2013; 15 (11): 942-947.

18. Wang RT, Zhang JR, Li Y, Liu T, Yu KJ. Neutrophil-Lymphocyte ratio is associated with arterial stiffness in diabetic retinopathy in type 2 diabetes. J Diabetes Complications 2015; 29 (2): 245-249.

19. Ilhan N, Daglioglu MC, Ilhan O et al. The Significance of the Neutrophil/Lymphocyte Ratio as a Simple Indicator of Inflammation in Age-related Macular Degeneration. Ocul Immunol Inflamm 2016; 24 (3): 362-363.

20. Kurtul BE, Ozer PA. The Relationship between Neutrophil-to-lymphocyte Ratio and Age-related Macular Degeneration. Korean J Ophthalmol 2016; 30 (5): 377-381.
21. Polat O, Yavaş GF, Inan S, Inan UU. Neutrophil-to-Lymphocyte Ratio as a Marker in Patients with Non-arteritic Anterior Ischemic Optic Neuropathy. Balkan Med J 2015; 32 (4): 382-387.

22. Guclu H, Ozal SA, Pelitli Gurlu V, Birgul R. Elevated Neutrophil Lymphocyte Ratio in Recurrent Optic Neuritis. J Ophthalmol 2015; 2015: 758687 .

23. Dursun A, Ozturk S, Yucel H et al. Association of neutrophil/lymphocyte ratio and retinal vein occlusion. Eur J Ophthalmol 2015; 25 (4): 343-346.

24. Karaca EE, Özmen MC, Ekici F, Yüksel E, Türkoğlu Z. Neutrophilto-lymphocyte ratio may predict progression in patients with keratoconus. Cornea 2014; 33 (11): 1168-1173.

25. Ozgonul C, Sertoglu E, Mumcuoglu T, Ozge G, Gokce G. Prediction of Pseudoexfoliation Syndrome and Pseudoexfoliation Glaucoma by Using Neutrophil to Lymphocyte Ratio and Platelet to Lymphocyte Ratio. Ocul Immunol Inflamm 2016; 24 (6): 665-670.

26. Ozgonul C, Sertoglu E, Mumcuoglu T, Kucukevcilioglu M. Neutrophil-to-Lymphocyte Ratio and Platelet-to-Lymphocyte Ratio as Novel Biomarkers of Primary Open-Angle Glaucoma. J Glaucoma 2016; 25 (10): e815-e820. 